\title{
The Cognitive Augmented Mobility Program (CAMP): Feasibility and Preliminary Efficacy
}

\section{Sunnybrook}

Katherine Dittmann ${ }^{1}$, Kay-Ann Allen ${ }^{1}$, Ashley Bergner ${ }^{2}$, Joyce Chen ${ }^{1,3}$, Elizabeth Inness ${ }^{4}$, Elizabeth Linkewich ${ }^{2,3,5}$, Sandra Pacione ${ }^{3}$, Jennifer Shaffer ${ }^{2}$, Ada Tang ${ }^{6}$, and Sara McEwen ${ }^{1,3}$ ${ }^{1}$ Sunnybrook Research Institute, ${ }^{2}$ Sunnybrook Health Sciences Centre, ${ }^{3}$ University of Toronto ${ }^{4}$ Toronto Rehab Institute, ${ }^{5}$ Toronto Stroke Networks, ${ }^{6}$ McMaster University

\section{Toronto Stroke}

\section{Networks}

\section{Purpose}

The objective of this project was to examine the feasibility and preliminary outcomes of an evidence-based cognitive augmented mobility program (CAMP) for persons with stroke.

\section{Relevance}

Current mobility treatment approaches for survivors of stroke are not usually associated with improved community participation, transfer of skills learned in therapy to new skills, nor skill maintenance ${ }^{1,2}$

A potential solution may be to combine the best practices for improving walking and mobility in persons with stroke with cognitive strategy training to improve problem solving, confidence, maintenance and transfer of skills to the community

Best evidence suggests therapists should incorporate task-specific training, aerobics, and strengthening to improve mobility in gait in persons with stroke

Related research suggests that incorporating cognitive problem-solving strategy training may lead to better skill maintenance and transfer ${ }^{3,4}$

\section{Methods}

Two groups of 4 participants post stroke, post usual care, with mobility goals, able to walk at least 3 metres, and able to follow directions.

\section{1 week pre-intervention}

\section{Pre-Intervention Session}

\section{- One-on-One with the} Physiotherapist (90min)

- Education regarding program, cognitive strategy, principles of exercise and best practice guidelines

- Develops self-selected goals with PT using COPM

- Review of cognitive strategy,

- Dynamic Performance Analysis (DPA)

- Discusses intervention outline based on goals

- Begin discussion re: transitions and post-discharge plans Goal-Plan-Do-Check (GPDC)

\section{Phase 1: Phase 2: Phase 3: Foundations (weeks 1-3) > Skill Acquisition (weeks 4-6) $>$ Consolidation (weeks 7-8) \\ Intervention}

\section{Group Education (5-10 minutes)}

- Provided at the beginning of each session includes education on: GPDC, exercise principles, task-specific strengthening, task-specific practice \& home activities; also allows for group discussion and questions

Warm-up (5 minutes) Full body warm-up, includes 30 second sit-to-stand
Individualized Exercise Program (IExP) (60-75 min)

- Task-specific program designed around selfselected goals

- Performed in a group setting set up in 4-6 taskspecific strength and task-specific practice stations

- GPDC \& guided discovery used throughout

- Progressive increase in level of independence and self-evaluation

\section{Goal Practice Session (within IExP)}

- Weekly 1:1 sessions with PT using \& teaching GPDC

- PT uses guided discovery instruction to support patient strategies and problem solving

- Provides opportunity to discuss \& modify the IExP and provide additional education

- GPDC framework used for goals developed for CAMP as well as for planning at home independently, and for setting new goals in the future after CAMP

\section{Cool-down (5-10 minutes)}

\section{Analysis \& Results}

Analysis: Due to the small sample size, non-parametric statistics were used. Medians and ranges were calculated, and to estimate efficacy, differences between assessment points were analyzed using Wilcoxon test and non-parametric effect size $r$ was calculated and were are coded as small (.1), medium (.3) or large (.5) effects. ${ }^{3}$ Additionally, for measures for which an estimate of a minimally important change was available, the proportion of participants who achieved that level was calculated and reported.

Table 1. Effect sizes \& Proportion of Cases Greater than Minimal Detectable Change (MDC) or Minimal Clinically Important Difference (MCID)

\begin{tabular}{|c|c|c|c|c|c|}
\hline \multirow[t]{2}{*}{ Outcome } & \multirow[t]{2}{*}{$\begin{array}{c}\text { MCID/ } \\
\text { MDC }\end{array}$} & \multicolumn{2}{|c|}{ Effect size $r$} & \multicolumn{2}{|c|}{$\begin{array}{l}\% \text { cases with change score } \\
\text { MDC or MCID }\end{array}$} \\
\hline & & $\mathrm{T} 1-\mathrm{T} 2$ & $\mathrm{~T} 1-\mathrm{T} 3$ & $\mathrm{~T} 1-\mathrm{T} 2$ & T1 - T3 \\
\hline Functional Independence Measure & MCID: $22^{5}$ & $0.38^{\mathrm{b}}$ & $0.25^{\mathrm{a}}$ & $0 \%$ & $0 \%$ \\
\hline 5 meter walk test $(\mathrm{m} / \mathrm{s})^{*}$ & MCID: $0.16 \mathrm{~m} / \mathrm{s}^{6}$ & $0.39^{\mathrm{b}}$ & $0.26^{a}$ & $50 \%$ & $50 \%$ \\
\hline 6 minute walk test $(\mathrm{m})$ & MCID: $34.4 m^{7}$ & $0.53^{\mathrm{c}}$ & $0.38^{b}$ & $37.5 \%$ & $37.5 \%$ \\
\hline Stroke Impact Scale - Participation & NA & $0.27^{\mathrm{a}}$ & 0.03 & - & - \\
\hline Stroke Impact Scale - Recovery & NA & $0.25^{\mathrm{a}}$ & $0.25^{\mathrm{a}}$ & - & - \\
\hline Activity-Specific Balance Confidence Scale & NA & $0.38^{\mathrm{b}}$ & 0.09 & - & - \\
\hline Berg Balance Scale & MDC: $4.7^{8,9}$ & $0.59^{\mathrm{c}}$ & $0.63^{c}$ & $62.5 \%$ & $62.5 \%$ \\
\hline Community Balance \& Mobility Scale & MDC: $8^{10}$ & $0.63^{c}$ & $0.63^{c \dagger}$ & $25 \%$ & $50 \%^{\dagger}$ \\
\hline $\begin{array}{l}\text { Canadian Occupational Performance Measure (COPM) - } \\
\text { Performance }\end{array}$ & MDC: $1.7^{11}$ & $0.63^{\mathrm{c}}$ & $0.59^{c}$ & $100 \%$ & $75 \%$ \\
\hline Canadian Occupational Performance Measure (COPM) - Satisfaction & MDC: $2.7^{11}$ & $0.63^{\mathrm{c}}$ & $0.60^{c}$ & $75 \%$ & $87.5 \%$ \\
\hline
\end{tabular}

\section{Discussion \& Conclusion}

The positive results included a large effect on self-selected goal performance at one-month follow-up and transfer of learning to balance and mobility skills

Findings suggest CAMP is feasible, and, that there is a potential complementary effect in combining best evidence for mobility and fitness for persons with stroke with cognitive strategy training that warrants further investigation

These results may be a precursor to future discussions exploring methods to include active problem-solving \& shared-decision making throughout care

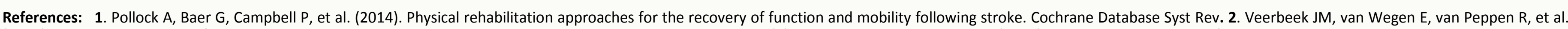

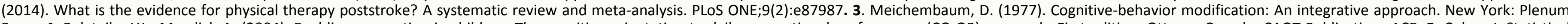

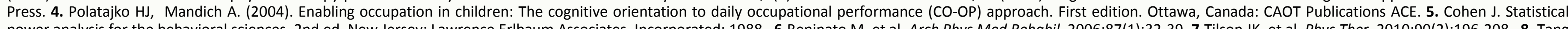

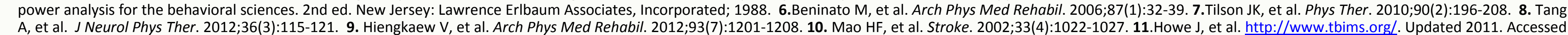

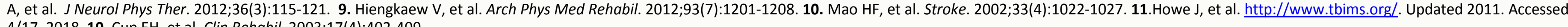
4/17, 2018. 10. Cup EH, et al. Clin Rehabil. 2003;17(4):402-409. 